\title{
Combining Multiple Matchers for Fingerprint Verification: A Case Study in FVC2004
}

\author{
J. Fierrez-Aguilar ${ }^{1, \star}$, Loris Nanni ${ }^{2}$, J. Ortega-Garcia ${ }^{1}$, \\ Raffaele Cappelli ${ }^{2}$, and Davide Maltoni ${ }^{2}$
}

1 ATVS, Escuela Politecnica Superior, Universidad Autonoma de Madrid, Avda. Francisco Tomas y Valiente 11, Campus de Cantoblanco, 28049 Madrid, Spain \{julian.fierrez, javier.ortega\}@uam.es

2 Biometric Systems Lab., DEIS, Universita di Bologna, Viale Risorgimento 2, 40136 Bologna, Italy \{lnanni, rcappelli, dmaltoni\}@deis.unibo.it

\begin{abstract}
Combining different algorithms submitted to the Third International Fingerprint Verification Competition (FVC2004) is studied. For this work, the matching results of more than 40 fingerprint systems from both academy and industry are available on standard benchmark data. In particular, we concentrate on score-level fusion of the different algorithms, studying the correlation between them by using featuresubset-selection techniques. Based on the general algorithmic descriptions provided by the participants, some interesting experimental findings are obtained.
\end{abstract}

\section{Introduction}

The increasing interest in a wide variety of practical applications for automatic personal identification and authentication has resulted in the popularity of biometric recognition systems [1. In particular, the use of fingerprint images has initiated much research, with a large number of different algorithmic approaches proposed during the last decades [2. As a result, recent efforts have been conducted in order to establish common evaluation scenarios enabling a fair comparison between competing systems [3]. In the case of fingerprint recognition, a series of International Fingerprint Verification Competitions (FVC) 4 ] have received great attention both from the academy and the industry. These competitions have provided common data and procedures widely available now for further research [2]. Other recent comparative benchmark studies include the Fingerprint Vendor Technology Evaluations organized by NIST [5].

On the other hand, one recent focus of interest in biometrics research is the successful combination of different sources of information resulting in the so-called multi-biometric approaches [6]. The general scheme of the multiple

\footnotetext{
* This work has been carried out while J. F.-A. was on a research visit at Bologna University.
} 


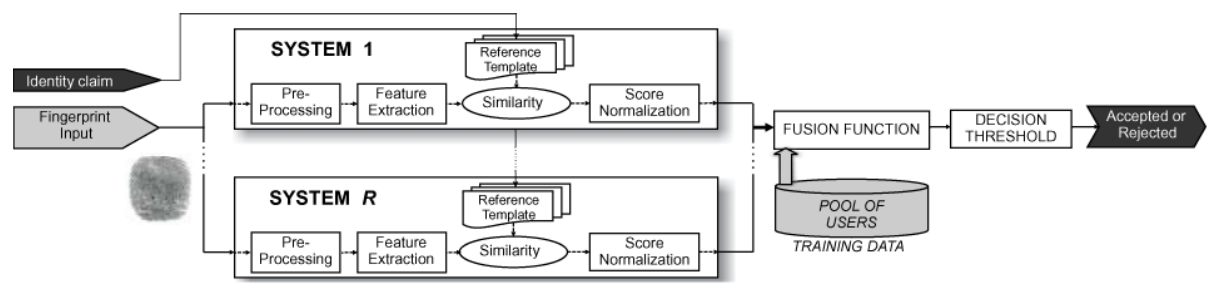

Fig. 1. System model of score-level multiple matcher approach for fingerprint verification

matcher approach that will be considered in the rest of the paper is depicted in Fig. 1 for the case of fingerprint verification.

The aim of this work is to study the combination of different fingerprint recognition systems submitted to FVC2004 and to analyze the benefits and limits of the resulting multiple matcher approaches. Based on the general algorithmic descriptions provided by most of the participants, and the experimental evidence obtained, we finally draw a number of conclusions of practical interest.

\section{FVC2004: Fingerprint Verification Competition}

Details of the competition and results were presented in [4] and more information appears in [7. Two different sub-competitions (open and light) were organized. Light category was intended for algorithms with restricted computing and memory usage, 26 algorithms participated in this case. 41 algorithms participated in the open category. In Table 1] we include general algorithmic descriptions of most of the systems, as provided by the participants, following the taxonomy in 2. Pointers to the identities of non-anonymous participants, individual results, and comparative charts can be found at [7. In this work, we focus on combining the algorithms competing in the open category.

Data for the competition consists of 4 different databases, the first three acquired with different sensors and the last of them created with a synthetic
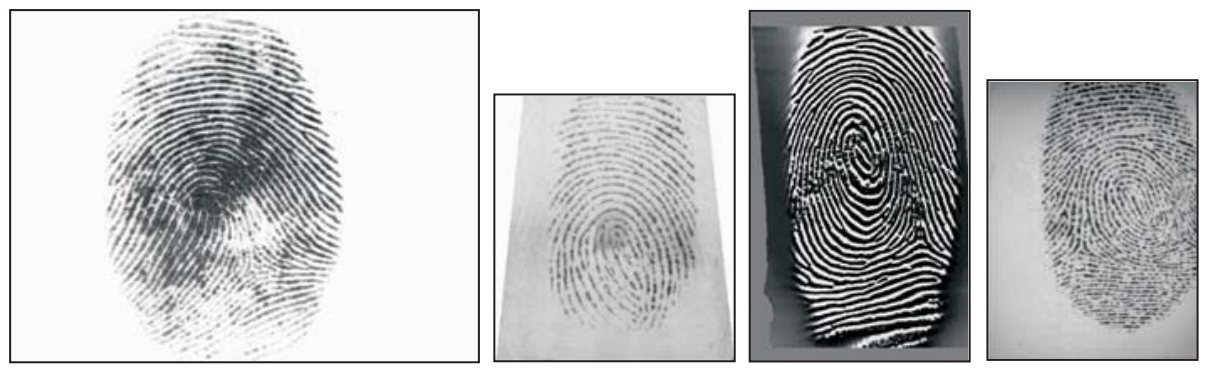

Fig. 2. Fingerprint examples from the four databases used in FVC2004 (left to right): DB1 (CrossMatch V300), DB2 (Digital Persona UareU 4000), DB3 (Atmel FingerChip), and DB4 (SFinGe v3.0). 
Table 1. High-level description of systems from 29 participants in FVC2004. Participant 071: only performs segmentation in the light category, alignment type is Displacement+Rotation in the light category and Non-linear in the open, raw Image parts and Correlation are used only in the open category. Participant 101: Segmentation is performed only on DB1.

\begin{tabular}{|c|c|c|c|c|c|c|c|c|c|c|c|c|c|c|c|c|c|}
\hline \multirow[b]{2}{*}{ 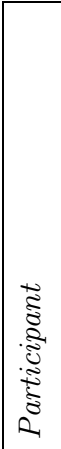 } & \multicolumn{2}{|c|}{ Preprocessing } & \multicolumn{2}{|c|}{ Alignment } & \multicolumn{8}{|c|}{ Features } & \multicolumn{5}{|c|}{ Matching } \\
\hline & 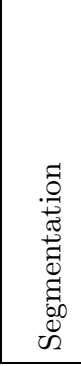 & 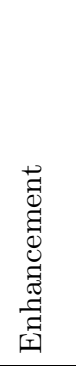 & 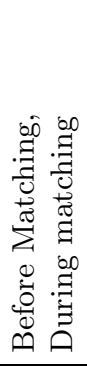 & 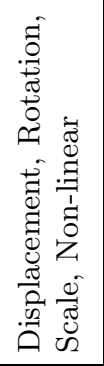 & 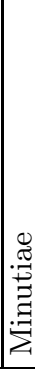 & 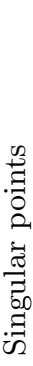 & $\begin{array}{l}0 \\
0 \\
0 \\
-1\end{array}$ & 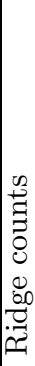 & 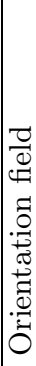 & 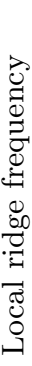 & 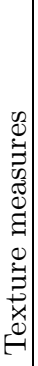 & 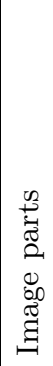 & 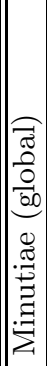 & 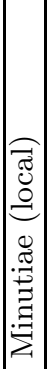 & 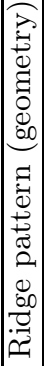 & 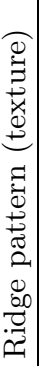 & 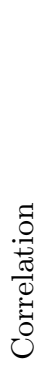 \\
\hline |002 & $\overline{\overline{\sqrt{ }}}$ & $\overline{\sqrt{ }}$ & $\overline{\bar{D}}$ & $\overline{\mathrm{NL}}$ & $\sqrt{ }$ & & & & $\sqrt{ }$ & & & & $\| \sqrt{ }$ & $\sqrt{ }$ & & & \\
\hline 009 & $\sqrt{ }$ & $\sqrt{ }$ & $\mathrm{BD}$ & $\overline{\text { DRS }}$ & $\sqrt{ }$ & $\sqrt{ }$ & $\sqrt{ }$ & $\sqrt{ }$ & $\sqrt{ }$ & $\sqrt{ }$ & & & $\sqrt{ }$ & & & & \\
\hline 016 & & $\sqrt{ }$ & & & $\sqrt{ }$ & & $\sqrt{ }$ & & & & & & $\sqrt{ }$ & & & & $\sqrt{ }$ \\
\hline 026 & & & & DR & $\sqrt{ }$ & & & $\sqrt{ }$ & $\sqrt{ }$ & & & & $\sqrt{ }$ & & & & \\
\hline 027 & $\sqrt{ }$ & $\sqrt{ }$ & $\mathrm{D}$ & DRS & & & & & & & $\sqrt{ }$ & & & & & $\sqrt{ }$ & $\sqrt{ }$ \\
\hline \begin{tabular}{|l||}
039 \\
\end{tabular} & $\sqrt{ }$ & $\sqrt{ }$ & $\mathrm{D}$ & $\mathrm{N}$ & $\sqrt{ }$ & & & & $\sqrt{ }$ & $\sqrt{ }$ & & & $\sqrt{ }$ & & & & \\
\hline \begin{tabular}{|l||}
041 \\
\end{tabular} & $\sqrt{ }$ & $\sqrt{ }$ & $\bar{D}$ & $\overline{\mathrm{DR}}$ & $\sqrt{ }$ & & $\sqrt{ }$ & & & & & & & & $\sqrt{ }$ & & \\
\hline 047 & $\sqrt{ }$ & & $\mathrm{D}$ & DRSN & $\sqrt{ }$ & $\sqrt{ }$ & $\sqrt{ }$ & $\sqrt{ }$ & $\sqrt{ }$ & $\sqrt{ }$ & $\sqrt{ }$ & $\sqrt{ }$ & $\sqrt{ }$ & $\sqrt{ }$ & & $\sqrt{ }$ & \\
\hline 049 & $\sqrt{ }$ & $\sqrt{ }$ & $\bar{D}$ & $\overline{\mathrm{DR}}$ & $\sqrt{ }$ & $\sqrt{ }$ & $\sqrt{ }$ & & $\sqrt{ }$ & $\sqrt{ }$ & & & $\sqrt{ }$ & $\sqrt{ }$ & & & \\
\hline 050 & $\sqrt{ }$ & $\sqrt{ }$ & $\mathrm{B}$ & DR & $\sqrt{ }$ & & & & $\sqrt{ }$ & & & & $\sqrt{ }$ & & & & \\
\hline \begin{tabular}{|l||}
051 \\
\end{tabular} & $\sqrt{ }$ & $\sqrt{ }$ & $\mathrm{D}$ & $\mathrm{DR}$ & $\sqrt{ }$ & $\sqrt{ }$ & & $\sqrt{ }$ & $\sqrt{ }$ & & & & $\sqrt{ }$ & $\sqrt{ }$ & & & $\sqrt{ }$ \\
\hline 067 & $\sqrt{ }$ & $\sqrt{ }$ & $\bar{D}$ & DRN & $\sqrt{ }$ & & & & $\sqrt{ }$ & & & & $\sqrt{ }$ & & & & \\
\hline \begin{tabular}{|l||}
068 \\
\end{tabular} & $\sqrt{ }$ & $\sqrt{ }$ & $\mathrm{D}$ & DR & $\sqrt{ }$ & & $\sqrt{ }$ & & & & & & $\sqrt{ }$ & & & & $\sqrt{ }$ \\
\hline \begin{tabular}{|l||}
071 \\
\end{tabular} & $(\sqrt{ })$ & $\sqrt{ }$ & $\mathrm{D}$ & $\mathrm{DR}(\mathrm{N})$ & $\sqrt{ }$ & $\sqrt{ }$ & $\sqrt{ }$ & $\sqrt{ }$ & $\sqrt{ }$ & & & $(\sqrt{ })$ & & $\sqrt{ }$ & $\sqrt{ }$ & & $(\sqrt{ })$ \\
\hline \begin{tabular}{|l|}
072 \\
\end{tabular} & $\sqrt{ }$ & $\sqrt{ }$ & $\bar{D}$ & $\overline{D R}$ & $\sqrt{ }$ & $\sqrt{ }$ & & & $\sqrt{ }$ & & & & $\sqrt{ }$ & & & & \\
\hline \begin{tabular}{|l||}
075 \\
\end{tabular} & $\sqrt{ }$ & $\sqrt{ }$ & $\bar{B}$ & DR & $\sqrt{ }$ & & & & & & & & & $\sqrt{ }$ & & & \\
\hline \begin{tabular}{|l||}
078 \\
\end{tabular} & $\sqrt{ }$ & $\sqrt{ }$ & $\mathrm{D}$ & DRS & $\sqrt{ }$ & & & & & & & & $\sqrt{ }$ & & & & \\
\hline 087 & $\sqrt{ }$ & $\sqrt{ }$ & $\mathrm{D}$ & DR & $\sqrt{ }$ & & & & $\sqrt{ }$ & $\sqrt{ }$ & $\sqrt{ }$ & & $\sqrt{ }$ & $\sqrt{ }$ & $\sqrt{ }$ & & \\
\hline \begin{tabular}{|l|}
097 \\
\end{tabular} & $\sqrt{ }$ & & $\mathrm{D}$ & DR & $\sqrt{ }$ & $\sqrt{ }$ & & & $\sqrt{ }$ & $\sqrt{ }$ & & & $\sqrt{ }$ & $\sqrt{ }$ & & & \\
\hline \begin{tabular}{|l||}
099 \\
\end{tabular} & $\sqrt{ }$ & $\sqrt{ }$ & $\bar{D}$ & DRN & $\sqrt{ }$ & & & & & & & & $\sqrt{ }$ & & & & \\
\hline \begin{tabular}{|l||}
101 \\
\end{tabular} & $(\sqrt{ })$ & $\sqrt{ }$ & $\mathrm{BD}$ & DRS & & $\sqrt{ }$ & $\sqrt{ }$ & $\sqrt{ }$ & $\sqrt{ }$ & $\sqrt{ }$ & & $\sqrt{ }$ & & & $\sqrt{ }$ & & $\sqrt{ }$ \\
\hline \begin{tabular}{|l||}
103 \\
\end{tabular} & $\sqrt{ }$ & $\sqrt{ }$ & & & $\sqrt{ }$ & & & & $\sqrt{ }$ & & & & & $\sqrt{ }$ & & & \\
\hline 104 & $\sqrt{ }$ & $\sqrt{ }$ & $\mathrm{D}$ & DR & $\sqrt{ }$ & & & & & & & & & $\sqrt{ }$ & & & \\
\hline \begin{tabular}{|l||}
105 \\
\end{tabular} & $\sqrt{ }$ & $\sqrt{ }$ & $\mathrm{B}$ & DR & $\sqrt{ }$ & $\sqrt{ }$ & & & $\sqrt{ }$ & & & & & $\sqrt{ }$ & $\sqrt{ }$ & & \\
\hline 106 & & & & & $\sqrt{ }$ & & $\sqrt{ }$ & & & & & & & $\sqrt{ }$ & & & \\
\hline 107 & & $\sqrt{ }$ & $\mathrm{D}$ & DRS & $\sqrt{ }$ & $\sqrt{ }$ & & & & & & & $\sqrt{ }$ & $\sqrt{ }$ & & & \\
\hline \begin{tabular}{|l||}
108 \\
\end{tabular} & $\sqrt{ }$ & $\sqrt{ }$ & $\mathrm{D}$ & DR & $\sqrt{ }$ & $\sqrt{ }$ & & & $\sqrt{ }$ & & & & & $\sqrt{ }$ & & & \\
\hline \begin{tabular}{|l||}
111 \\
\end{tabular} & $\sqrt{ }$ & $\sqrt{ }$ & $\mathrm{D}$ & $\overline{D R}$ & $\sqrt{ }$ & & & & $\sqrt{ }$ & & & & $\sqrt{ }$ & & & & \\
\hline \begin{tabular}{|l||}
113 \\
\end{tabular} & $\sqrt{ }$ & $\sqrt{ }$ & $\overline{\mathrm{D}}$ & $\mathrm{N}$ & $\sqrt{ }$ & $\sqrt{ }$ & $v$ & & $\sqrt{ }$ & $v$ & & $\sqrt{ }$ & $\sqrt{ }$ & $\sqrt{ }$ & & & $\sqrt{ }$ \\
\hline
\end{tabular}


generator, see Fig. 2 for example images. Worth noting, the image quality is low to medium due to exaggerated plastic distortions, and artificial dryness and moistness [4]. Each database comprises 100 different fingers with 8 impressions per finger. Different fingers were used for different databases.

The experimental protocol was as follows. The 8 impressions of each finger were matched against each other avoiding symmetric matches, thus generating $(100 \times 8 \times 7) / 2=2800$ genuine matching scores. The first impression of each user was also matched against the first impression of every other user avoiding symmetric matches, thus generating $(100 \times 99) / 2=4950$ impostor matching scores. The matching scores are similarity values ranging between 0 and 1 . A number of performance indicators were then obtained including score histograms, verification error rates, computing time, memory allocated, and others [4]. In this work we carry out comparative experiments focusing on the Equal Error Rate between individual and combined algorithms.

\section{Methods}

\subsection{Score Fusion Methods}

Three different combination strategies are evaluated in the present work.

Sum. This basic fusion method consists in averaging the matching scores provided by the different systems. Under some mild statistical assumptions [89, this simple method is demonstrated to give good results for the biometric authentication problem.

SVM. Another popular approach to combining multiple matchers in biometric recognition is to treat the combination stage as a second-level pattern recognition problem on the matching scores that are to be fused [10], and then use standard learning paradigms in order to obtain combining functions. Comparative studies exist in this regard [1], favoring the Support Vector Machine-based fusion approach over others [12. Linear and RBF kernels are used in the present work [13].

Dempster-Shafer. Another group of methods well adapted to multiple classifier approaches is based on evidence accumulation [14. From this group, we have selected and implemented the fusion scheme based on Dempster-Shafer theory as described in [14].

\subsection{Feature Subset Selection Method}

In order to study the effects of the correlation between different systems on the performance of their combination, we have considered the fusion stage as a second-level 2-class pattern recognition problem. The similarity score output of each system is seen as a different feature, and the two classes correspond to impostor and genuine attempts, respectively.

Best fingerprint systems to combine are then chosen by running Sequential Forward Floating Selection (SFFS) [15] on the similarity scores available from 
the competition. Goodness of the feature subset was defined as the empirical EER obtained using the sum rule.

\section{Experiments}

\subsection{Experimental Protocol}

Feature selection and fusion experiments are carried out separately for the 4 different databases considered. Whenever training was needed for the fusion schemes (i.e., SVM and Dempster-Shafer), 2-fold cross-validation was used, dividing the available scores in two partitions (different fingers in each of them).

\subsection{Results}

A comparative chart is depicted in Fig. 3 for the case of combining 2, 4, and 6 fingerprint systems (horizontal axis of each subplot) with all the fusion strategies considered (bars with different greyscale), on the 4 databases considered

Table 2. Systems chosen with SFFS. EER in \%

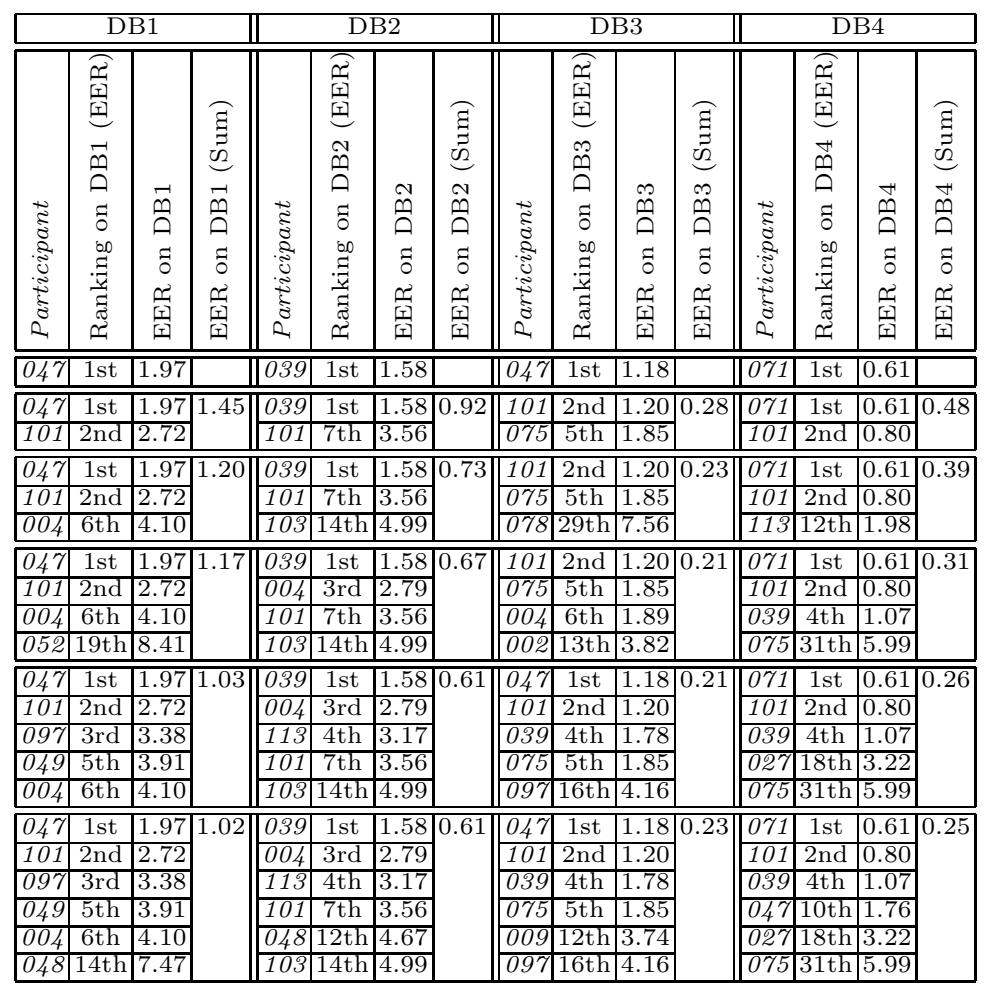



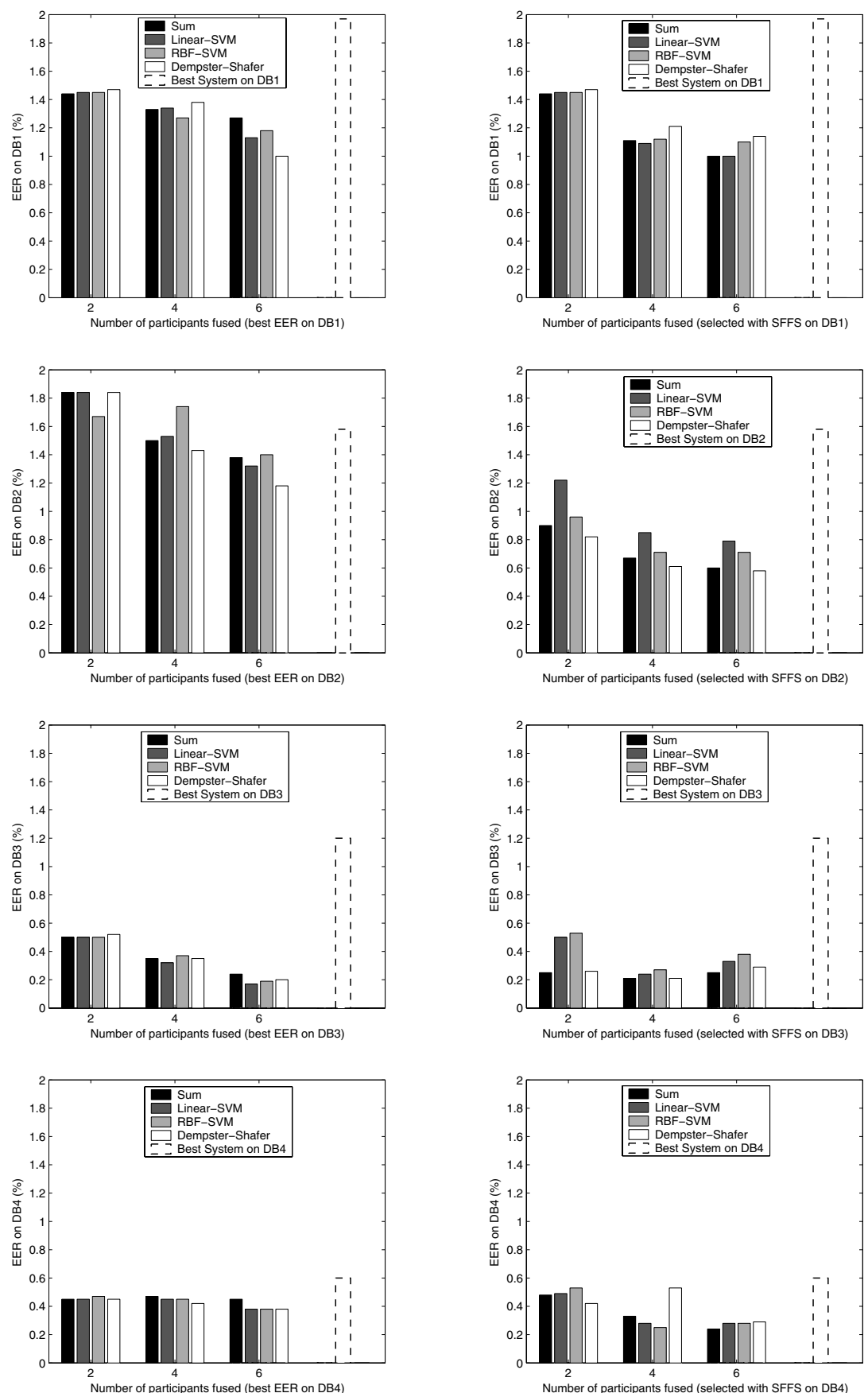

Fig. 3. Results. Open Category. 


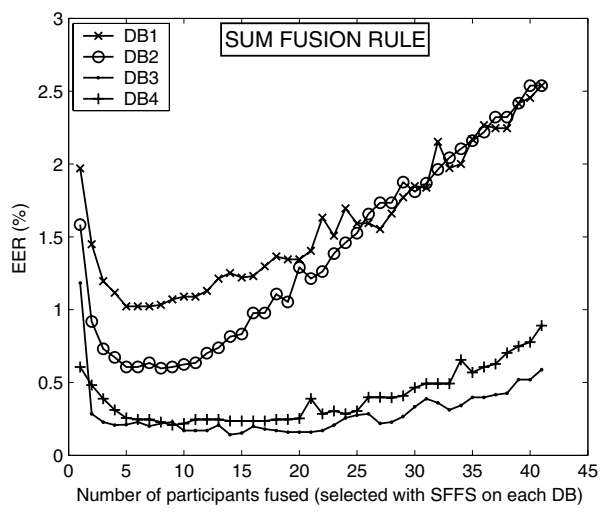

Fig. 4. Verification performance results for an increasing number of fingerprint systems selected with SFFS for fusion

(different rows), either combining the best individual systems on each database (left column) or selecting the best combination through SFFS (right column). The performance of the best individual system in each database is also shown as a dashed bar for reference. Substantial performance improvements are obtained when combining different systems in most of the cases, specially DB3. Also interesting is the fact that combining systems chosen with SFFS always leads to improved performance and this is not the case when combining best individual systems (e.g., DB2). Also interestingly, no particular fusion method seems to consistently outperform the others.

In Fig. 4 we plot the results of the combination of an increasing number of systems selected with SFFS for the 4 databases considered. The performance improves with the fusion of up to 5 to 7 systems and then deteriorates when combining more than 10 systems. The systems automatically selected by the SFFS algorithm are shown in Table2, following the system numbering of Table1.

\section{Discussion and Conclusions}

A number of experimental findings can be extracted from the results. Firstly, the simple fusion approach based on sum rule is not clearly outperformed by more complex trained fusion approaches. Secondly, the combination of the top performing individual systems can be outperformed by other combinations (e.g., the best 2-system combination on DB3 is obtained by fusing the systems individually ranked as $2 \mathrm{nd}$ and $5 \mathrm{th}$, see Table 2). Analyzing various individual cases, best combinations are usually obtained when combining systems that are based on heterogenous matching strategies, such as minutia-based with ridge[16] and/or correlation-based [17.

Also worth noting, the maximum performance is found when combining a small number of systems (about 7 in this case study). Initially, the performance 
improvement is significantly high, but as we increase the number of systems to fuse the performance stabilizes reaching a maximum. If more systems are combined, the performance actually degrades.

\section{Acknowledgements}

This work has been supported by Spanish TIC2003-08382-C05-01, Italian 2004098034, and European IST-2002-507634 projects. Authors also thank Prof. Anil K. Jain for helpful comments. J. F.-A. is supported by a FPI scholarship from Comunidad de Madrid.

\section{References}

1. Jain, A.K., Ross, A., Prabhakar, S.: An introduction to biometric recognition. IEEE Trans. on Circuits and Systems for Video Technology 14 (2004) 4-20

2. Maltoni, D., Maio, D., Jain, A.K., Prabhakar, S.: Handbook of Fingerprint Recognition. Springer (2003)

3. Wayman, J., Jain, A., Maltoni, D., Maio, D., eds.: Biometric Systems: Technology, Design and Performance Evaluation. Springer (2005)

4. Maio, D., Maltoni, D., et al.: FVC2004: Third Fingerprint Verification Competition. In: Proc. ICBA, Springer LNCS-3072 (2004) 1-7

5. Wilson, C., et al.: FpVTE2003: Fingerprint Vendor Technology Evaluation 2003 (NISTIR 7123) website: http://fpvte.nist.gov/.

6. Jain, A.K., Ross, A.: Multibiometric systems. Communications of the ACM 47 (2004) 34-40

7. FVC2004 website: http://bias.csr.unibo.it/fvc2004.

8. Bigun, E.S., et al.: Expert conciliation for multi modal person authentication systems by Bayesian statistics. In: Proc. AVBPA, LNCS-1206 (1997) 291-300

9. Kittler, J., Hatef, M., Duin, R., Matas, J.: On combining classifiers. IEEE Trans. on Pattern Anal. and Machine Intell. 20 (1998) 226-239

10. Verlinde, P., Chollet, G., Acheroy, M.: Multi-modal identity verification using expert fusion. Information Fusion 1 (2000) 17-33

11. Ben-Yacoub, S., Abdeljaoued, Y., Mayoraz, E.: Fusion of face and speech data for person identity verification. IEEE Trans. on Neural Networks 10 (1999) 1065-1074

12. Gutschoven, B., Verlinde, P.: Multi-modal identity verification using support vector machines (SVM). In: Proc. FUSION, IEEE Press (2000) 3-8

13. Fierrez-Aguilar, J., et al.: Exploiting general knowledge in user-dependent fusion strategies for multimodal biometric verification. In: Proc. ICASSP (2004) 617-620

14. Kuncheva, L.I., Bezdek, J.C., Duin, R.P.W.: Decision templates for multiple classifier fusion: An experimental comparison. Pattern Recogn. Lett. 34 (2001) 299-314

15. Jain, A., Zongker, D.: Feature selection: Evaluation, application, and small sample performance. IEEE Trans. on Pattern Anal. and Machine Intell. 19 (1997) 153-158

16. Ross, A., Jain, A.K., Reisman, J.: A hybrid fingerprint matcher. Pattern Recognition 36 (2003) 1661-1673

17. Nandakumar, K., Jain, A.K.: Local correlation-based fingerprint matching. In: Proc. Indian Conf. on Comp. Vision, Graphics and Image Process. (2004) 503-508 